\title{
Direct probes of dark matter in the cluster 1ES0657-556 through microwave observations
}

\author{
S. Colafrancesco ${ }^{1}$, P. de Bernardis ${ }^{2}$, S. Masi ${ }^{2}$, G. Polenta ${ }^{2}$, and P. Ullio ${ }^{3}$ \\ 1 INAF - Osservatorio Astronomico di Roma, via Frascati 33, 00040 Monteporzio, Italy \\ e-mail: cola@mporzio.astro.it \\ 2 Dipartimento di Fisica, Università Roma 1, P.le A. Moro 2, Roma, Italy \\ 3 Scuola Internazionale Superiore di Studi Avanzati, via Beirut 2-4, 34014 Trieste, Italy
}

Received 14 October 2006 / Accepted 25 January 2007

\section{ABSTRACT}

\begin{abstract}
Aims. The cluster 1ES0657-556 is an ideal astrophysical laboratory to study the distribution and the nature of Dark Matter because this last component is spatially separated from the intracluster gas. We show that microwave observations can provide crucial probes of Dark Matter in this system.

Methods. We calculate the expected SZ effect from Dark Matter annihilation in the main mass concentrations of the cluster 1ES0657-556, and we estimate the sources of contamination, confusion and bias to asses its significance.

Results. We find that SZ observations at $v \approx 223 \mathrm{GHz}$ can resolve both spatially and spectrally the $\mathrm{SZ}_{\mathrm{DM}}$ signal and isolate it from the other SZ signals, and mainly from the thermal SZ effect which is null at $v \sim 220-223 \mathrm{GHz}$ for the case of 1ES0657-556. We conclude that SZ observations with $\lesssim$ arcmin resolution and $\lesssim \mu \mathrm{K}$ sensitivity of 1ES0657-556 are crucial, and maybe unique, to find direct astrophysical probes of the existence and of the nature of Dark Matter, or to set strong experimental limits.
\end{abstract}

Key words. dark matter - galaxies: clusters: individual: 1ES0657-556 - galaxies: clusters: general - cosmic microwave background

\section{Introduction}

Dark Matter (DM) annihilations in the halo of galaxies and galaxy clusters have crucial astrophysical implications. In fact, if $\mathrm{DM}$ is constituted by weakly interacting massive particles (for which the leading candidate is the lightest supersymmetric particle, plausibly the neutralino $\chi$ ), their annihilation produces secondary particles (e.g., neutral and charged pions, secondary electrons and protons, neutrinos) that give rise to various astrophysical signals. These are, among others, observable fluxes of positrons, antiprotons, gamma rays, neutrinos, as well as signals due to secondary electrons which cover the whole e.m. spectrum (see, e.g., Colafrancesco et al. 2006, for details): synchrotron radio emission (in the intra-cluster magnetic field), bremsstrahlung emission (if there is co-spatial intra-cluster gas), inverse Compton emission due to the up-scattering of CMB photons and hence a specific SZ effect (as first noticed and derived by Colafrancesco 2004). The spatial and spectral intensity of the astrophysical signals coming from $\chi \chi$ annihilation is expected, however, to be confused or even overcome by other astrophysical signals originating from the intracluster (IC) gas and/or from the relativistic plasmas present in the cluster atmospheres, especially when all these components are co-spatially distributed with the DM component. This situation occurs in most galaxy clusters (see discussion by Colafrancesco et al. 2006 for the case of Coma).

An ideal system to detect DM annihilation signals would, therefore, be a cluster with a clear spatial separation between the various matter components. This is, indeed, the case of the cluster 1ES0657-556 where the spatial distribution of DM is clearly offset w.r.t. that of the IC gas (Clowe et al. 2006).
The two baryonic clumps of hot gas emit X-rays by (thermal) bremsstrahlung, as observed by Chandra (Markevitch et al. $2002,2004)$. The shock observed in the western-most region of the cluster (Markevitch et al. 2002) might be the site of high energy emission from particles accelerated at the shock. Hard X-ray emission from the direction of 1ES0657-556 has been marginally detected by Rossi-XTE (Petrosian et al. 2006) but its angular resolution is not sufficient to give any information on the spatial distribution of this emission. No gamma-ray emission has been detected from this system with EGRET. The extended radio halo associated to this cluster (Liang et al. 2000) has a surface brightness slightly elongated along the direction of the two X-ray clumps, but without clear signatures of radiobrightness enhancements at the DM clump locations. Finally, the SZ maps of 1ES0657-556 obtained with ACBAR (with $\sim 4.5$ arcmin FWHM resolution, Gomez et al. 2003) are quite smooth and regular with no evidence of enhancement at both $\mathrm{X}$-ray and/or DM clump locations. In this Letter, we will compute the specific feature of the SZ effect (herefater SZE) produced by $\mathrm{DM}$ annihilation, $\mathrm{SZ}_{\mathrm{DM}}$, in the cluster 1ES0657-556 and we will show that it is possible to detect such $\mathrm{SZ}_{\mathrm{DM}}$ signal with a specific observational strategy. The relevant physical quantities are calculated using $H_{0}=70 \mathrm{~km} \mathrm{~s}^{-1} \mathrm{Mpc}^{-1}$ and a flat, $\Lambda \mathrm{CDM}\left(\Omega_{\mathrm{m}}=0.3, \Omega_{\Lambda}=0.7\right)$ cosmological model.

\section{The complex SZ effect in 1ES0657-556}

The various SZ signals expected from the subsystems of the cluster 1ES0657-556 are: i) the $\mathrm{SZ}_{\mathrm{DM}}$ effect, which is expected to be located at the two DM clumps; ii) the thermal SZ effect $\left(\mathrm{SZ}_{\mathrm{th}}\right)$ which is expected to be located at the two X-ray clumps. 
We will compute in the following these two sources of SZE and we will also discuss the possible sources of contamination, bias and confusion. The general expression for the SZE which is valid in the Thomson limit for a generic electron population in the relativistic limit and includes also the effects of multiple scatterings and the combination with other electron population in the cluster atmospheres has been derived by Colafrancesco et al. (2003). This approach is the one that will be used for the derivation of the $\mathrm{SZ}_{\mathrm{DM}}$ effect induced by the secondary electrons produced by $\chi \chi$ annihilation (see the original derivation by Colafrancesco 2004 ) as well as for the $\mathrm{SZ}_{\text {th }}$ produced by the hot X-ray emitting gas. According to these results, the spectral distortion of the CMB spectrum induced by a population of electron with momentum distribution $f_{\mathrm{e}}(p)$ can be written as

$\Delta I(x)=2 \frac{\left(k_{\mathrm{B}} T_{0}\right)^{3}}{(h c)^{2}} \tilde{y} \tilde{g}(x)$,

where $T_{0}$ is the CMB temperature and

$\tilde{y}=\frac{\sigma_{T}}{m_{\mathrm{e}} c^{2}} \int P_{\mathrm{e}} \mathrm{d} \ell$

in terms of the pressure $P_{\mathrm{e}}$ contributed by the specific electron population. The spectral function $\tilde{g}(x)$, with $x \equiv h v / k_{\mathrm{B}} T_{0}$, can be written as

$\tilde{g}(x)=\frac{m_{\mathrm{e}} c^{2}}{\langle\epsilon\rangle}\left\{\frac{1}{\tau}\left[\int_{-\infty}^{+\infty} i_{0}\left(x \mathrm{e}^{-s}\right) P(s) \mathrm{d} s-i_{0}(x)\right]\right\}$

in terms of the photon redistribution function $P(s)$ and of $i_{0}(x)=$ $2\left(k_{\mathrm{B}} T_{0}\right)^{3} /(h c)^{2} \cdot x^{3} /\left(\mathrm{e}^{x}-1\right)$. Here $\tau=\int \mathrm{d} \ell n_{\mathrm{e}}$ is the optical depth of the electrons with number density $n_{\mathrm{e}}$, and $\langle\epsilon\rangle \equiv$ $\frac{\sigma_{\mathrm{T}}}{\tau} \int P_{\mathrm{e}} \mathrm{d} \ell=\int_{0}^{\infty} \mathrm{d} p f_{\mathrm{e}}(p) \frac{1}{3} p v(p) m_{\mathrm{e}} c$ is the average energy of the electronic plasma. The photon redistribution function $P(s)=$ $\int \mathrm{d} p f_{\mathrm{e}}(p) P_{\mathrm{s}}(s ; p)$ with $s=\ln \left(v^{\prime} / v\right)$, in terms of the CMB photon frequency increase factor $v^{\prime} / v$, depends on the electron momentum distribution $f_{\mathrm{e}}(p)$, where the momentum $p$ is normalized to $m_{\mathrm{e}} c$. The CMB temperature change produced by the SZE is finally given by

$\frac{\Delta T}{T_{0}}=\frac{\left(\mathrm{e}^{x}-1\right)^{2}}{x^{4} \mathrm{e}^{x}} \frac{\Delta I}{I_{0}}$.

The specific $\mathrm{SZ}_{\mathrm{DM}}$ and $\mathrm{SZ}_{\mathrm{th}}$ effects for the various electronic components in the cluster 1ES0657-556 are computed following the approach previously described.

\subsection{The $S Z_{D M}$ effect in the cluster 1ES0657-556}

The calculation of the secondary electron spectrum from $\chi \chi$ annihilation in galaxy clusters has been already presented in details by Colafrancesco \& Mele (2001) and Colafrancesco et al. (2006), and here we will only recall the relevant steps necessary for the present purpouses.

We assume, for simplicity, a spherical DM halo model for each DM clump of the cluster 1ES0657-556, as indicated by the lensing maps derived by Clowe et al. (2006), with DM density profile given by $g(x)=x^{-\eta}(1+x)^{\eta-\xi}$, with $x \equiv r / r_{\mathrm{s}}$. Values $\eta=1$ and $\xi=3$ reproduce the Navarro et al. (1997) density profile.

The neutralino number density profiles $n_{\chi}(E, r)=$ $n_{\chi, 0}(E) g(r)$ of the two DM clumps have been calculated following the approach described in Colafrancesco et al. (2006) with a NFW DM density profile and the following structure parameters: $M_{\mathrm{vir}}=10^{15} M_{\odot}, R_{\mathrm{vir}}=1.97 \mathrm{Mpc}$ and $c_{\mathrm{vir}}=R_{\mathrm{vir}} / r_{\mathrm{s}}=5.66$ (for the larger East DM clump); $M_{\mathrm{vir}}=6.25 \times 10^{13} M_{\odot}, R_{\mathrm{vir}}=$ $0.784 \mathrm{Mpc}$ and $c_{\mathrm{vir}}=7.56$ (for the "bullet" West DM clump).

The $\chi$ annihilation rate in the DM clumps is $R=n_{\chi}(r)\langle\sigma v\rangle_{0}$, where $\langle\sigma v\rangle_{0}$ is the $\chi \chi$ annihilation cross section averaged over a thermal velocity distribution at freeze-out temperature. The range of neutralino masses and pair annihilation cross sections in the most general supersymmetric DM setup is extremely wide (see discussion in Colafrancesco et al. 2006, 2007). We consider here, specifically, the neutralino models worked out in Colafrancesco et al. (2006) with $M_{\chi}=20,40$ and $81 \mathrm{GeV}$ and with their specific values of $\langle\sigma v\rangle_{0}$.

The electron source functions $Q_{\mathrm{e}}(E, r) \propto\langle\sigma v\rangle_{0} n_{\chi}^{2}(E, r)$ for the specific neutralino model considered here have been derived in Colafrancesco et al. (2006) and the time evolution of the electron spectrum is given by the equation

$\frac{\partial n_{\mathrm{e}}(E, r)}{\partial t}-\frac{\partial}{\partial E}\left[n_{\mathrm{e}}(E, r) b(E)\right]=Q_{\mathrm{e}}(E, r)$,

where spatial diffusion can be safely neglected in cluster-size DM clumps (Colafrancesco et al. 2006). The function

$$
\begin{aligned}
b(E)= & b_{\mathrm{IC}}^{0}\left(\frac{E}{\mathrm{GeV}}\right)^{2}+b_{\mathrm{syn}}^{0} B_{\mu}^{2}\left(\frac{E}{\mathrm{GeV}}\right)^{2} \\
& +b_{\mathrm{Coul}}^{0} n_{\mathrm{th}}\left(1+\log \left(\gamma / n_{\mathrm{th}}\right) / 75\right) \\
& +b_{\mathrm{brem}}^{0} n_{\mathrm{th}}\left(\log \left(\gamma / n_{\mathrm{th}}\right)+0.36\right)
\end{aligned}
$$

gives the energy loss per unit time at energy $E$ where $n_{\text {th }}$ is the mean number density of thermal electrons in $\mathrm{cm}^{-3}, \gamma \equiv E / m_{\mathrm{e}} c^{2}$ and $b_{\mathrm{IC}}^{0} \simeq 0.25, b_{\mathrm{syn}}^{0} \simeq 0.0254, b_{\mathrm{Coul}}^{0} \simeq 6.13, b_{\mathrm{brem}}^{0} \simeq 1.51$, all in units of $10^{-16} \mathrm{GeV} \mathrm{s}^{-1}$. The equilibrium spectrum $n_{\mathrm{e}}(E, r)$ obtained solving Eq. (5) allows to calculate the $\mathrm{SZ}_{\mathrm{DM}}$ effect. Figure 1 shows the CMB temperature change $\Delta T$ evaluated at the centers of the two DM clumps for different values of $M_{\chi}$. The $\mathrm{SZ}_{\mathrm{DM}}$ signals are overcome by the $\mathrm{SZ}_{\mathrm{th}}$ signals at low $(v \lesssim$ $200 \mathrm{GHz})$ and high $(v \gtrsim 230 \mathrm{GHz})$ frequencies, while they dominate in the frequency range where the zero of $\mathrm{SZ}_{\text {th }}$ is found, i.e. at $v \approx 223 \mathrm{GHz}$ for the East clump and at $v \approx 219 \mathrm{GHz}$ for the West clump. At such frequencies the $\mathrm{SZ}_{\mathrm{DM}}$ temperature decrement takes values $\approx-21.1,-10.6,-0.3 \mu \mathrm{K}$ (East clump) and values $\approx-8.4,-4.2,-0.1 \mu \mathrm{K}$ (West clump) for $M_{\chi}=20,40,81 \mathrm{GeV}$, respectively.

\subsection{The $S Z_{t h}$ effect in the cluster $1 E S 0657-556$}

The $\mathrm{SZ}_{\mathrm{th}}$ from the two X-ray clumps of the cluster 1ES0657-556 is also computed from the general approach delineated in Eqs. (1)-(4). Following Markevitch et al. (2002, 2004) and Tucker et al. (1998), we use the simplifying assumption that the IC gas distribution can be described by isothermal spheres fitted by a $\beta$-profile. For the East X-ray clump we adopt $k T_{\mathrm{e}}=14 \mathrm{keV}$ (Markevitch et al. 2002, 2004) and a $\beta$-profile with $r_{\mathrm{c}}=257 \mathrm{kpc}$, $\beta=0.62$ and a central IC gas density $n_{\mathrm{th}, 0}=6.71 \times 10^{-3} \mathrm{~cm}^{-3}$ (Tucker et al. 1998). For the West X-ray clump we adopt $k T_{\mathrm{e}}=$ $6 \mathrm{keV}$ and a $\beta$-profile with $r_{\mathrm{c}}=125 \mathrm{kpc}, \beta=0.7$ and a central IC gas density $n_{\mathrm{th}, 0}=2 \times 10^{-2} \mathrm{~cm}^{-3}$, neglecting the additional central power-law density profile induced by the presence of the shock (Markevitch et al. 2002, 2004). Thus, SZ th $_{\text {from this last }}$ $\mathrm{X}$-ray clump should be considered as slightly underestimated.

The possible non-equilibrium and non-thermal effects induced by the merging shock are not crucial for the aims of this paper since the shock is located outside the two X-ray clumps and at a large angular distance from the two DM clumps (see 

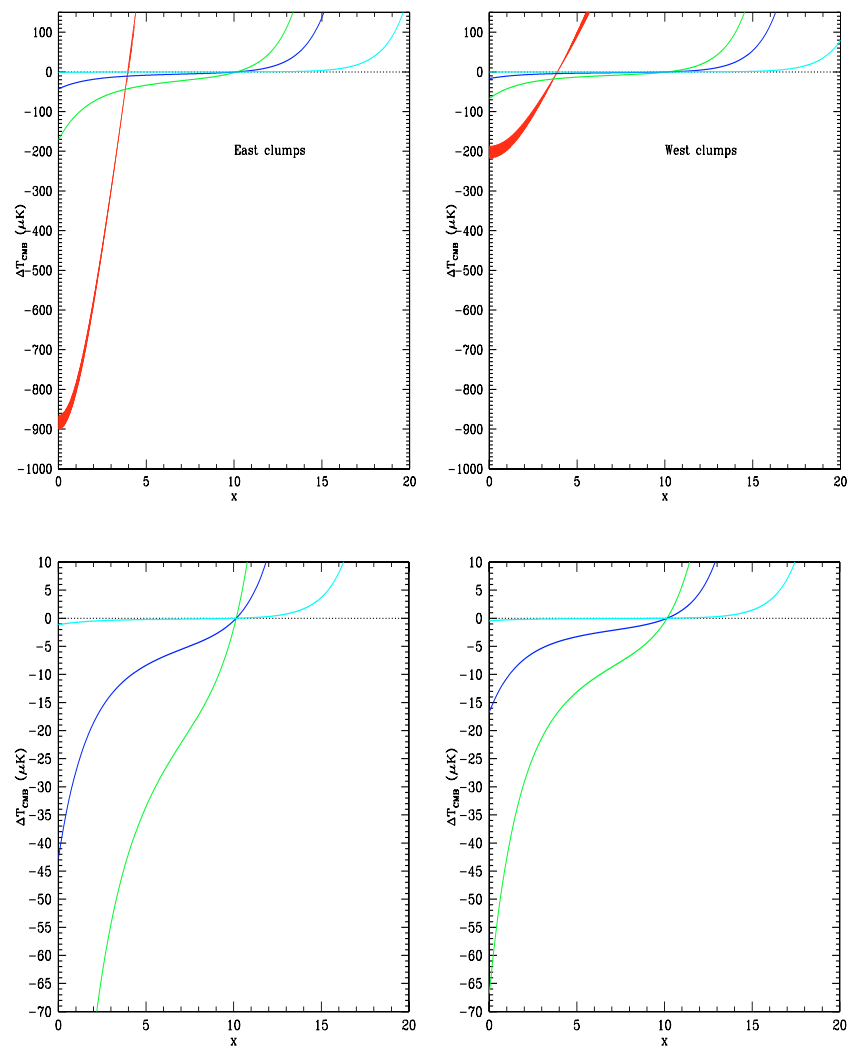

Fig. 1. The CMB temperature decrement produced by the East (left panels) and West (right panels) clumps of gas (red shaded areas which take into account the effect of IC gas temperature uncertainties) and DM with neutralino mass $M_{\chi}=20 \mathrm{GeV}$ (green), $M_{\chi}=40 \mathrm{GeV}$ (blue) and $M_{\chi}=81 \mathrm{GeV}$ (cyan). The lower panels show a zoom of the $\mathrm{SZ}_{\mathrm{DM}}$ effect only.

Markevitch et al. 2002, 2004). Possible non-thermal SZE produced by shocks is, however, expected to be at most at a level of a few $\mu \mathrm{K}$ (see Colafrancesco et al. 2003), and therefore it could be, at most, a source of bias for the thermal SZE at the location of the $\mathrm{X}$-ray clumps. The $\mathrm{SZ}_{\text {th }}$ temperature change for the two $X$-ray clumps is also shown in Fig. 1. A major difference between the $\mathrm{SZ}_{\mathrm{th}}$ and $\mathrm{SZ}_{\mathrm{DM}}$ spectral functions is the different position of the zero of the SZE which is moved to higher frequencies in the case of the DM produced electrons with respect to the case of the thermal distribution. As a consequence, the $\mathrm{SZ}_{\mathrm{DM}}$ effect appears as a negative contribution to the overall SZE at all the frequencies which are relevant for the SZ experiments, $x \sim 0.5-10$.

\subsection{Contamination, bias and confusion}

Possible sources of confusion for the microwave search for DM in 1ES0657-556 are: CMB anisotropy, emission of the IC medium, emission of galaxies and AGNs along the line of sight, thermal and kinematic SZE from the X-ray clumps. For the estimates of the foreground contamination we consider as peaks of the DM distribution producing SZE the regions included in the $\kappa=0.3$ contour of Fig. 1 in Clowe et al. (2006). These are roughly $1.3^{\prime}$ in diameter, and are separated by $\sim 4.8^{\prime}$.

The measurement we propose must be carried out with an instrument with angular resolution of $\sim 1^{\prime} F W H M$, able to resolve the two $\mathrm{SZ}_{\mathrm{DM}}$ peaks, and to distinguish them from the thermal and kinematic SZE of the two X-ray clumps. Larger scales are not significant for the measurement, and must be removed either in the measurement or in the data analysis. This will remove most of the CMB anisotropy. In fact, integrating over the power spectrum of CMB anisotropy best fitting current measurements (including lensing effects), we find that an angular highpass filter removing scales $\gtrsim 3^{\prime}$ will cut multipoles $\ell \lessgtr 3600$, leaving a residual rms fluctuation of primary $\mathrm{CMB}$ anisotropy of $0.8 \mu \mathrm{K} \mathrm{rms}$. This is further reduced by the finite angular resolution of the telescope.

In the two $\mathrm{SZ}_{\mathrm{DM}}$ peaks we find 14 galaxies in the area of the East peak and 6 galaxies in the area of the West peak (see Barrena et al. 2002). To obtain an estimate of the mm-wave emission of these galaxies, we take the mm-wave spectrum of an "average", "normal" galaxy (like M99) and redshift it to $z=0.3$. Then we compute the flux in the bands centered at 90, 145, $217,270,345,545 \mathrm{GHz}$ and we find the total flux by multiplying it by the number of galaxies counted in the West and East $\mathrm{SZ}_{\mathrm{DM}}$ peaks. In the West peak, we obtain a total flux of 0.2, 0.4, 1.0, 1.6, 3.4, $19 \mathrm{mJy}$, respectively, in the bands quoted above. These signals correspond to a CMB anisotropy of 4.0, 4.8, 8.0, 13,40 , and $760 \mu \mathrm{K}$ for a $1^{\prime} F W H M$ beam. These are comparable in size to expected $\mathrm{SZ}_{\mathrm{DM}}$ signal, at least in the 90,145 , and $217 \mathrm{GHz}$ bands, but are positive signals, while the $\mathrm{SZ}_{\mathrm{DM}}$ signal is negative at the same frequencies. The same holds for AGNs: there is only one known radio source (SUMSS J065837-555718) listed in the NED database in the area of the East peak, and none in the area of the West peak. This source has been observed with the MOST radio telescope, and features a power law SED, with slope $\alpha \sim-0.9$. If the same SED is used to extrapolate to the frequencies of interest here, the total flux is safely negligible with respect to the expected $\mathrm{SZ}_{\mathrm{DM}}$ in all the bands considered here: for a $1^{\prime} F W H M$ beam the signal is below $1 \mu \mathrm{K}$ in the 145,217 , 270 and $345 \mathrm{GHz}$ bands.

Unresolved background galaxies produce a flux with positive and negative fluctuations with respect to the average. For a $1^{\prime}$ beam at $220 \mathrm{GHz}$, we get a rms signal of $\sim 3 \mu \mathrm{K}$, estimated using the model of Lagache et al. (2004).

The $\mathrm{SZ}_{\mathrm{th}}$ emission from the IC gas is offset with respect to the DM peaks, so that only the tail of the signal is present in the $\mathrm{SZ}_{\mathrm{DM}}$ peaks. The $\mathrm{SZ}_{\mathrm{th}}$ emission is certainly affected by the complex temperature distribution of the X-ray clumps (see Markevitch et al. 2002) producing fluctuations in the SZ maps (since $\left.\Delta T \propto \int \mathrm{d} \ell n_{\mathrm{e}} T_{\mathrm{e}}\right)$. This makes the maps at low $(150 \mathrm{GHz})$ and high $(350 \mathrm{GHz})$ frequency shown in Fig. 2 as only indicative of the observable SZ signal. However, we stress that the $\mathrm{SZ}$ maps at $v \approx 219-223 \mathrm{GHz}$ are quite realistic because at 223 (219) GHz for the East (West) X-ray peak, the $\mathrm{SZ}_{\text {th }}$ is null, and temperature uncertainties do not sensitively alter this result (see Fig. 1): this is, henceforth, the privileged band for this measurement.

The $\left(\Delta T / T_{0}\right)_{\text {kin }}$ at the clump centers is $\$ 2.7 \times$ $10^{-6}\left(\tau / 10^{-3}\right)\left(V_{\mathrm{p}} / 10^{3} \mathrm{~km} \mathrm{~s}^{-1}\right)$ and is maximum at $217 \mathrm{GHz}$. Small values of $\Delta T_{\text {kin }}$ are found at the location of the two DM clumps because: i) the optical depth of the secondary electrons is small §a few $10^{-5}$; ii) the optical depth of the residual IC gas at the DM peak locations is small §a few $10^{-4}$, and iii) the peculiar velocity of the X-ray clumps and DM clumps along the line of sight is relatively small (Markevitch 2004), since the major merging event occurs almost completely on the plane of the sky, i.e. mostly perpendicular to the line of sight. Even an extreme value $V_{\mathrm{p}} \approx 600 \mathrm{~km} \mathrm{~s}^{-1}$ (well above the rms value $\approx 300 \pm 80 \mathrm{~km} \mathrm{~s}^{-1}$ found for galaxy clusters, see Giovanelli et al. 1998) would produce a total $\Delta T_{\text {kin }} \sim 0.4 \mu \mathrm{K}$ $(\sim 0.2 \mu \mathrm{K})$ in the East (West) DM clump. In conclusion, being 

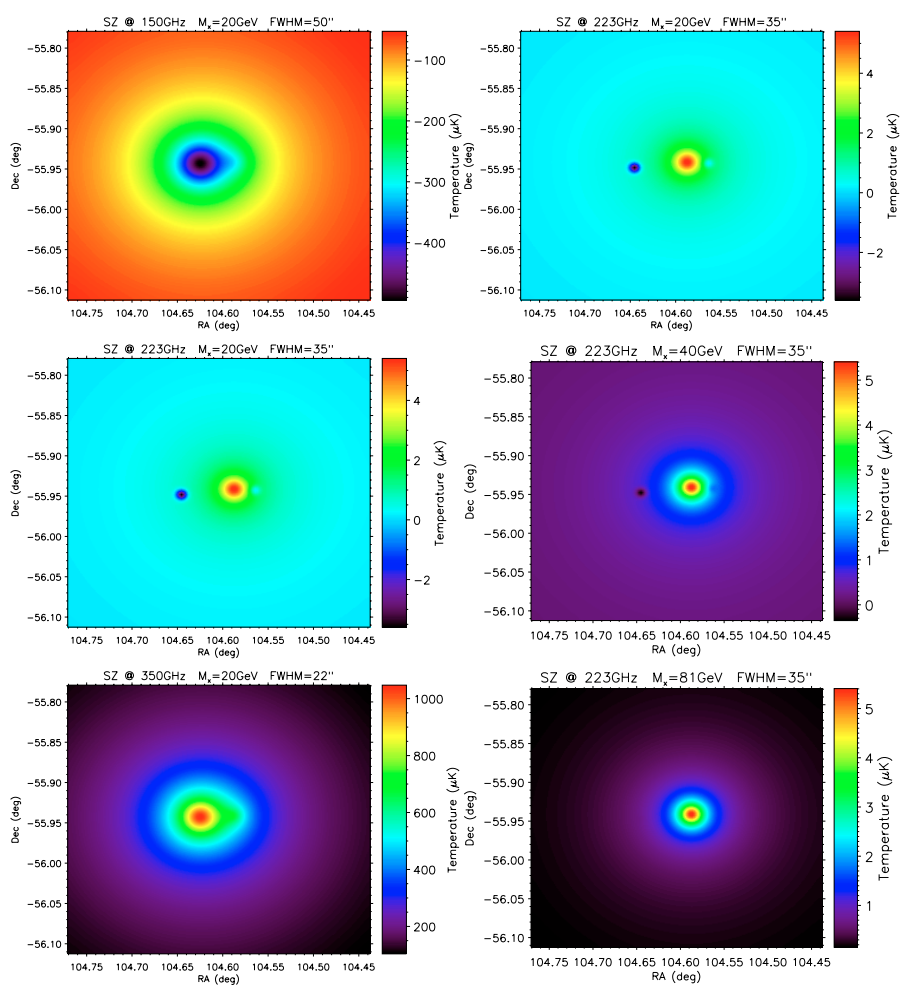

Fig. 2. Left: simulated SZ maps of the cluster 1ES0657-556 as observable with the SPT at $v=150 \mathrm{GHz}$ (upper panel), $v=223 \mathrm{GHz}$ (mid panel), $v=350 \mathrm{GHz}$ (lower panel). A value $M_{\chi}=20 \mathrm{GeV}$ has been adopted here. Right: simulated SZ maps of the cluster 1ES0657-556 as observable with the SPT at $v=223 \mathrm{GHz}$ for three different $\chi$ masses: $M_{\chi}=20 \mathrm{GeV}$ (upper panel), $40 \mathrm{GeV}$ (mid panel) and $81 \mathrm{GeV}$ (lower panel).

the two DM peaks offset with respect to the $\mathrm{SZ}_{\text {th }}$ peaks, the kinetic SZ effect is at most a marginal residual signal at the location of the two DM peaks of the cluster 1ES0657.

In these conditions the detectability of $\mathrm{SZ}_{\mathrm{DM}}$ is limited mainly by the fluctuations of the unresolved galaxies background. Negative or positive fluctuations can be found at the locations of the $\mathrm{SZ}_{\mathrm{DM}}$ peaks, affecting its detection. From realistic scan simulations at $220 \mathrm{GHz}$ with a $1^{\prime} F W H M$ beam, we estimate that a $2-\sigma(3-\sigma)$ detection has a likelihood $\gtrsim 95 \%$ if the $\mathrm{SZ}_{\mathrm{DM}}$ in the beam is $\gtrsim 6.5 \mu \mathrm{K}(\gtrsim 10 \mu \mathrm{K})$. These limits are comparable to the expected $\mathrm{SZ}_{\mathrm{DM}}$ signal estimated in Sect. 2.1. Complementary observations at higher and lower frequencies should confirm the nature of the signal, which in the case of the unresolved galaxies increases strongly with frequency, while is basically constant for the $\mathrm{SZ}_{\mathrm{DM}}$ There are several instruments expected to provide SZ surveys from the southern hemisphere in the near future. The 10-m South Pole Telescope (SPT, Ruhl et al. 2004) will exploit the excellent environmental conditions of the antarctic winter to produce deep surveys of SZ clusters at 90, 150, 220 and $270 \mathrm{GHz}$. With a $F W H M$ of $\lesssim 1^{\prime}$ this instrument is ideally suited to perform the DM search outlined here. The sensitivity of the SZ survey will be $\sim 10 \mu \mathrm{K}$ for each $1^{\prime}$ pixel at $150 \mathrm{GHz}$ : we suggest deeper observations for 1E0657-558. A sensitivity of $\sim 0.5 \mu \mathrm{K}$ per $1^{\prime}$ pixel at $150 \mathrm{GHz}$ can be obtained in 4 days of integration. Increasing atmospheric and bolometer noise prevents reaching the same sensitivity in the 220 and $270 \mathrm{GHz}$ bands (see also Gomez et al. 2003; Runyan et al. 2003 for the case of experiments working at larger angular scales). Still, the sensitivity should be good enough to see the two $\sim 10 \mu \mathrm{K}$ negative signals of $\mathrm{SZ}_{\mathrm{DM}}$. Similar considerations apply to the Atacama Cosmology Telescope (Kosowsky 2003), to the APEX bolometer arrays (Lee et al. 2004) and to the BOLOCAM instrument at the CSO (Glenn et al. 1998). Figure 2 shows the CMB maps expected from an observation of 1ES0657-556 at $v=150,223$ and $350 \mathrm{GHz}$ (with bandwidths of $\approx 25 \%$ ) with the SPT. The $\mathrm{SZ}_{\mathrm{DM}}$ effect clearly emerges localized at the two DM clumps for $v=223 \mathrm{GHz}$ where $\mathrm{SZ}_{\text {th }}$ vanishes for the brighter $\mathrm{X}$-ray clump with $k T_{\mathrm{e}}=14 \mathrm{keV}$. This is the optimal frequency to detect the $\mathrm{SZ}_{\mathrm{DM}}$ from the cluster 1ES0657-556. Such an experiment is able to set strong constraints on DM particle mass in the range $M_{\chi} \sim 20-50 \mathrm{GeV}$, while the SZ signals for $M_{\chi} \gtrsim 80 \mathrm{GeV}$ is unobservable. Higher frequency measurements are needed to monitor foreground contamination. Observations of 1E0657-558 with the OLIMPO experiment (Masi et al. 2005), using bolometer arrays at 145, 217, 345 and $545 \mathrm{GHz}$, with beam FWHM of $3.3^{\prime}, 2.2^{\prime}, 2.2^{\prime}, 2.2^{\prime}$ respectively, will be able to resolve the two SZE peaks, and will not be hampered by atmospheric fluctuations even in the highest frequency bands. In $10 \mathrm{~h}$ of integration over the cluster area, the sensitivity of OLIMPO in the 217 (345) GHz band will be $\approx 1.5$ (3.0) $\mu \mathrm{K}$ per pixel (with a pixel side of $\left.1^{\prime}\right)$. These measurements will nicely complement those of the ground based instruments, allowing an effective separation of the different astrophysical components, with special sensitivity to the fluctuating background from unresolved galaxies. An exciting perspective is the upgrade of a space-borne telescope with a Fourier Transform Spectrometer. This can provide the combination of high throughput and spectral resolution needed to compare the $\mathrm{SZ}_{\mathrm{th}}$ null to the surrounding frequencies, thus providing convincing evidence to distinguish between the different signal components (see Fig. 1). A first test can be carried out already with the OLIMPO experiment, preparing the way for a future satellite mission.

\section{Conclusions}

The $\mathrm{SZ}_{\mathrm{DM}}$ effect is an inevitable consequence of the presence and of the nature of DM in large-scale structures. Its analysis in the special case of 1ES0657-556 can provide a direct physical probe for the presence and for the nature of DM in cosmic structures.

How these observations compare to other possible DM signal detections? In general, both gamma-ray and radio observations of DM annihilation are quite powerful to set constraints on the neutralino mass and composition (see, e.g. Colafrancesco et al. 2006, 2007). However, in the case of 1ES0657-556 the expected gamma-ray emission associated to the DM clumps is too low ( $\$ 1$ count vs. $\sim 10$ background counts at $E>1 \mathrm{GeV}$ ) and cannot be resolved by GLAST from other possible sources of gamma-ray emission, both from the cluster 1ES0657-556 and from AGNs in the field. In addition, the GLAST spatial resolution ( 9-18 arcmin at 10 and $1 \mathrm{GeV}$, respectively) cannot provide any clear spatial separation between the DM gammaray signals (expected to be concentrated at the DM clumps) and other possible gamma-ray signals originating within the atmosphere of 1ES0657-556. Radio telescopes have, in principle, excellent resolution and sensitivity to probe the different spectra and brightness distribution of the DM-induced synchrotron emission. For the sake of illustration, we evaluated that the DM-induced synchrotron emission from the East DM clump is 3-10 mJy (for a smooth or smooth plus 50\% mass clumpiness NFW DM profile, $M_{\chi}=40 \mathrm{GeV},\langle\sigma v\rangle_{0}=4.7 \times 10^{-25} \mathrm{~cm}^{3} \mathrm{~s}^{-1}$ model used by Colafrancesco et al. 2006 for Coma, with a $1 \mu \mathrm{G}$ magnetic field) at $v=100 \mathrm{MHz}$, still marginally detectable by 
LOFAR. Theoretical uncertainties associated to the amplitude of the magnetic field in the DM clumps of 1ES0657-556 render, however, the prediction of the expected signals quite uncertain.

In such a context, the possible detection of the $\mathrm{SZ}_{\mathrm{DM}}$ effect will provide an important complementary, and maybe unique, probe of the nature of DM.

Acknowledgements. The author thanks the Referee for useful comments and suggestions.

\section{References}

Barrena, R., Biviano, A., Ramella, M., Falco, E. E., \& Seitz, S. 2002, A\&A, 386, 816

Clowe, et al. 2006, preprint [arXiv: astro-ph/0608407]

Colafrancesco, S. 2004, A\&A, 422, L23

Colafrancesco, S., \& Mele, B. 2001, ApJ, 562, 24

Colafrancesco, S., Marchegiani, P., \& Palladino, E. 2003, A\&A, 397, 27
Colafrancesco, S., Profumo, S., \& Ullio, P. 2006, A\&A, 455, 21

Colafrancesco, S., Profumo, S., \& Ullio, P. 2007, PRD, in press

Giovanelli, R., Haynes, M. P., Salzer, J. J., et al. 1998, AJ, 116, 2632

Glenn, J., et al. 1998, Proc. SPIE 3357, 326, Advanced Technology MMW, Radio, and Terahertz Telescopes

Gomez, P., Romer, A. K., \& Peterson, J. B. 2003 [arXiv:astro-ph/0311263]

Kosowsky, A. 2003, New Astron. Rev., 47, 939

Lagache, G., Dole, H., \& Puget, J. L. 2004, ApJS, 154, 112

Lee, A., et al., http://bolo.berkeley.edu/apexsz/instrument.html

Liang, H., Hunstead, R. W., Birkinshaw, M., \& Andreani, P. 2000, ApJ, 544, 686

Markevitch, M., Gonzalez, A. H., David, L., et al. 2002, ApJ, 567, L27

Markevitch, M., Gonzalez, A. H., Clowe, D., et al. 2004, ApJ, 606, 819

Masi, S., Calvo, M., Conversi, L., et al. 2005, in Proc. of the Intern. School of Physics E. Fermi, Course CLIX, ed. F. Melchiorri, \& Y. Rephaeli, SIF, IOS press, 359

Navarro, J., Frenk, C., \& White, S. D. M. 1997, ApJ, 490, 493

Petrosian, V., Madejski, G., \& Luli, K. 2006, ApJ, 652, 948

Ruhl, J., Ade, P. A. R., Carlstrom, J. E., et al. 2004, Proc. SPIE, 5498, 11

Runyan, M. C., Ade, P. A. R., Bock, J. J., et al. 2003, New Astron. Rev., 47, 915 